\title{
Einfluss der Korrosion auf die Schirmdämpfung versilberter Gestricke und Gewebe
}

\author{
M. Tasser and G. Wehnert \\ Georg-Simon-Ohm Fachhochschule Nürnberg, Lehrgebiet Makromolekulare Chemie, Keßlerplatz 12, 90489 Nürnberg, \\ Deutschland
}

Zusammenfassung. Zur Abschirmung elektromagnetischer Strahlung werden unter anderem metallisierte Gestricke eingesetzt, die versilbertes Polyamid enthalten.

Solche Materialien finden beispielsweise Anwendungen als flexible Verpackungen oder als Strumpfmaterialien im medizinischen Bereich, z.B. bei der Therapie des Phantomschmerzes.

Versilbertes Polyamid ist dem korrosiven Angriff schwefelhaltiger Verbindungen ausgesetzt, welche die Schirmdämpfungswirkung aufgrund der Ausbildung von Silbersulfidschichten herabsetzen.

Untersucht wurde, inwieweit Silbersulfidbildung die Schirmdämpfung beeinträchtigt und ob die Silbersulfidbildung durch Schutzschichten aus Titandioxid $\left(\mathrm{TiO}_{2}\right)$ verhindert werden kann.

Die Silbersulfidschichten wurden mit Hilfe einer alkalischen Natriumsulfid-Lösung (Tuccillo-Nielsen-Lösung) hergestellt. Titandioxid-Schichten wurden durch ein Sol-GelVerfahren abgeschieden.

Die untersuchten versilberten Gestricke zeigten im Bereich von $300 \mathrm{MHz}$ bis $4 \mathrm{GHz}$ eine weitgehend konstante Schirmdämpfung von ca. $40 \mathrm{~dB}$, abhängig von der Strickart. Durch Belegung der Oberfläche mit Silbersulfid nahm die Schirmdämpfung auf ca. 5-10 dB ab. Dünne, durch Sol-GelVerfahren abgeschiedene $\mathrm{TiO}_{2}$-Schichten verhinderten nicht die Ausbildung von Silbersulfidschichten.

Durch Reduktion des Silbersulfids mit Aluminium in Natriumchlorid-Lösung konnte die Schirmdämpfung teilweise wiederhergestellt werden, was sich an einem Anstieg der Schirmdämpfung auf ca. $25 \mathrm{~dB}$ zeigte.

Correspondence to: M. Tasser (m.tasser@web.de) G. Wehnert (gerd.wehnert@fh-nuernberg.de)

\section{Einleitung}

Zur Abschirmung hochfrequenter elektro-magnetischer Strahlung werden unter anderem metallisierte Gestricke und Gewebe eingesetzt, die versilbertes Polyamid enthalten. Solche Materialien dienen z.B. zum Schutz der Flugkörper von Lenkwaffen. Eine neuere Anwendung ist die medizinische Behandlung von Patienten, die nach einer Amputation unter Phantomschmerzen leiden. Momentan gibt es auf dem Markt so genannte Liner, die über den Stumpf gezogen werden. Sie bestehen unter anderem aus versilbertem Polyamid-Garn und werden je nach Anwendung entweder direkt auf der Haut getragen oder in Silikon eingebettet.

Silber neigt zum Ausbilden sulfidischer Schichten (,,Anlaufen"). Silbersulfid ist kein metallischer Leiter. Die Dämpfung elektromagnetischer Strahlung könnte durch Silbersulfidschichten beeinträchtigt werden.

\section{Versilbertes Polyamid}

Polyamid kann im Schmelzspinnverfahren zu dünnen Fäden extrudiert werden, die beispielsweise in der Strumpfindustrie Anwendung finden. Auf Polyamid-Fäden lässt sich nach Bekeimung mit Palladium galvanisch Silber abscheiden. Dabei kann es zur Ausbildung von Fehlstellen kommen (Abb. 1). Die Aufnahmen wurden mit einem Rasterelektronenmikroskop (REM, Leitz AMR 1000, Leitz, Wetzlar) angefertigt.

\section{Silbersulfid}

Eine der auffälligsten Eigenschaften metallischen Silbers $(\mathrm{Ag})$ ist die Empfindlichkeit gegenüber Schwefelwasserstoff $\left(\mathrm{H}_{2} \mathrm{~S}\right)$. Ungeschützte Silberoberflächen verfärben sich unter schwefelwasserstoffhaltiger Atmosphäre in Gegenwart von Feuchtigkeit bereits nach einigen Stunden. Nach zwei Tagen bis einer Woche tritt eine blauschwarze, nach drei Wochen

Published by Copernicus Publications on behalf of the URSI Landesausschuss in der Bundesrepublik Deutschland e.V. 

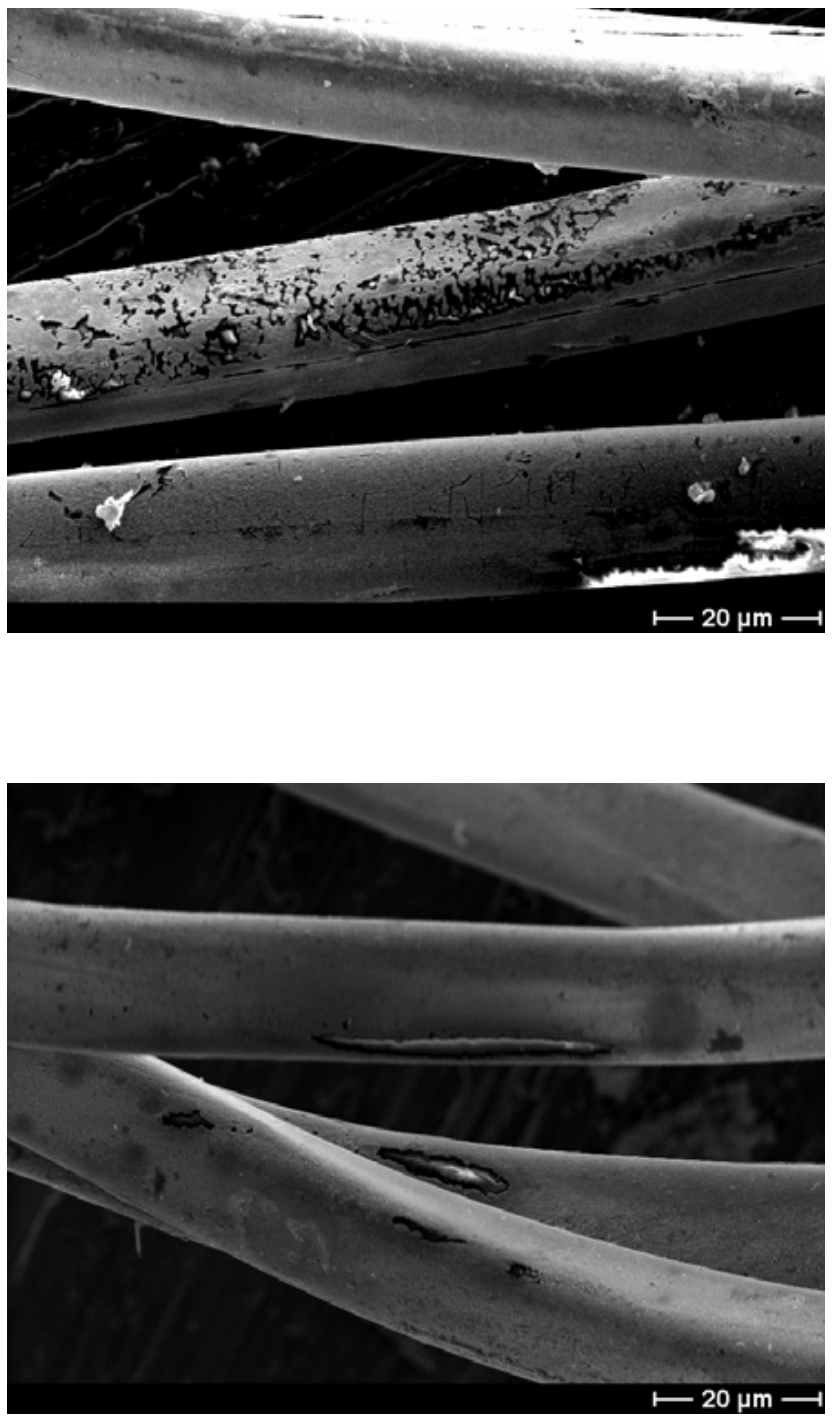

Abb. 1. Versilbertes Polyamid mit Fehlstellen.

eine tiefschwarze Silbersulfidoberfläche $\left(\mathrm{Ag}_{2} \mathrm{~S}\right)$ auf. Die Bildung von Silbersulfid $\left(\mathrm{Ag}_{2} \mathrm{~S}\right)$ wird durch folgende Gleichung beschrieben.

$2 \mathrm{Ag}+\mathrm{H}_{2} \mathrm{~S}+\frac{1}{2} \mathrm{O}_{2} \rightarrow \mathrm{Ag}_{2} \mathrm{~S}+\mathrm{H}_{2} \mathrm{O}$

Unterschiedliche Versuche haben gezeigt, dass Silber bei Ausschluss von Luftsauerstoff nicht von $\mathrm{H}_{2} \mathrm{~S}$ angegriffen wird und eine Entwicklung von $\mathrm{H}_{2}$ aus $\mathrm{H}_{2} \mathrm{~S}$ nicht stattfindet (Gmelins Handbuch der anorganischen Chemie, 1971).

Kim (2003) untersuchte den Einfluss von ppm-Gehalten an Stickstoffdioxid und Schwe-felwasserstoff sowie von adsorbiertem Wasser auf die Korrosion von Silber mittels einer Quarzkristallmikrowaage. In Abb. 2 ist die Massenzunahme in Abhängigkeit von der Zeit, in der die mit Silber beschich-

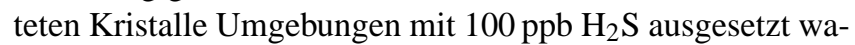
ren, zu sehen. Es wurde in Atmosphären mit 75\% und 15\%

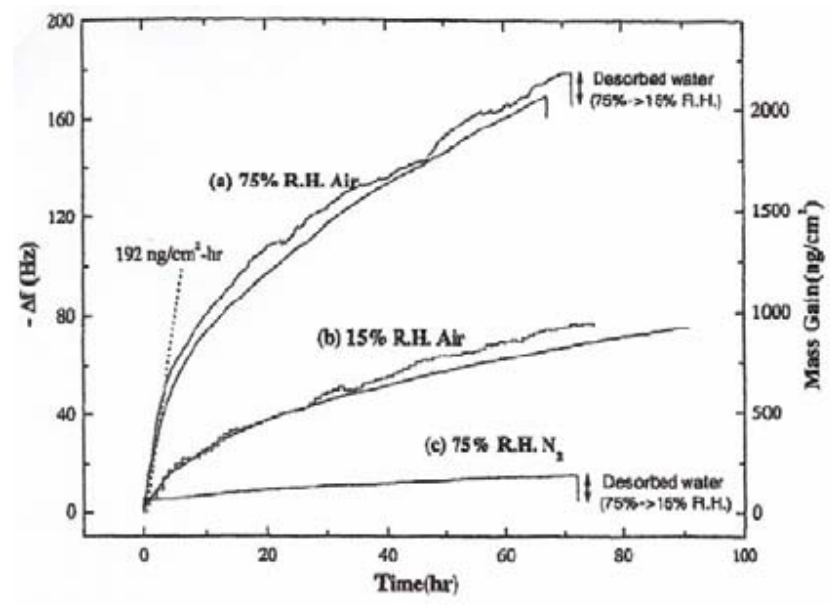

Abb. 2. Massenzunahme durch $\mathrm{Ag}_{2} \mathrm{~S}$-Bildung mit der atmosphärischen Zusammensetzung als Parameter (Kim, 2003).

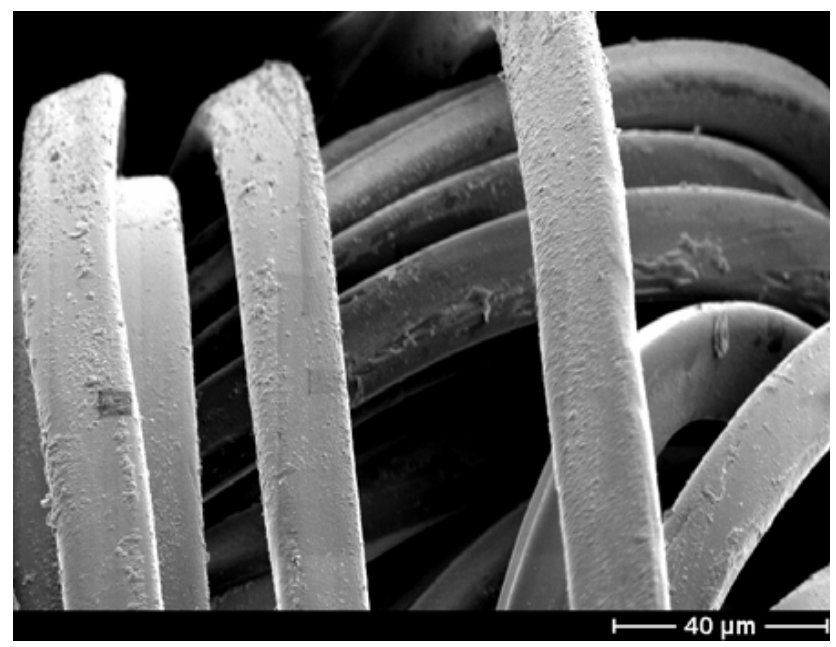

Abb. 3. REM Aufnahmen von $\mathrm{Ag}_{2} \mathrm{~S}$ Schichten auf versilberten Polyamid-Garn.

Raumfeuchte sowie 75\% Raumfeuchte in Stickstoff gemessen.

Insgesamt ist festzustellen, dass der Anlaufprozess in $\mathrm{H}_{2} \mathrm{~S}$ Umgebung sowie in $\mathrm{NO}_{2}$-Umgebung vom Sauerstoff und von der Luftfeuchtigkeit abhängt (Kim, 2003).

$\mathrm{Ag}_{2} \mathrm{~S}$ kommt in 4 Modifikationen vor. Unter normalem Druck entstehen bei steigender Temperatur nacheinander $\alpha$ $\mathrm{Ag}_{2} \mathrm{~S}, \beta-\mathrm{Ag}_{2} \mathrm{~S}, \gamma-\mathrm{Ag}_{2} \mathrm{~S}$, bei hohem Druck $\delta-\mathrm{Ag}_{2} \mathrm{~S}$. Das bei Raumtemperatur beständige $\alpha-\mathrm{Ag}_{2} \mathrm{~S}$ (Akanthit) kristallisiert monoklin und geht bei $175-180^{\circ} \mathrm{C}$ in das kubisch innenzentrierte $\beta-\mathrm{Ag}_{2} \mathrm{~S}$ (Argentit) über. Ab einer Temperatur von $600^{\circ} \mathrm{C}$ ändert sich die Modifikation in das kubisch flächenzentrierte $\gamma-\mathrm{Ag}_{2} \mathrm{~S}$.

Wird Silber bei Raumtemperatur an Luft gelagert, so entstehen innerhalb einiger Tage Oberflächenfilme von $\alpha-\mathrm{Ag}_{2} \mathrm{~S}$. Der Oberflächenfilm besteht anfangs aus fast sphärischen 

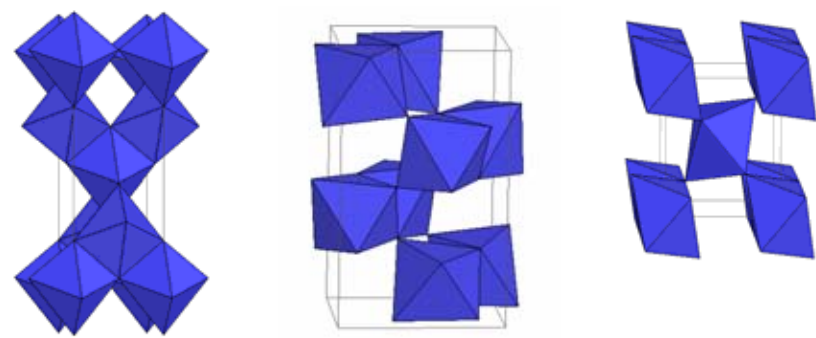

Abb. 4. Links: Anatas, mittig: Brookit, rechts: Rutil (Jostan, 1985).

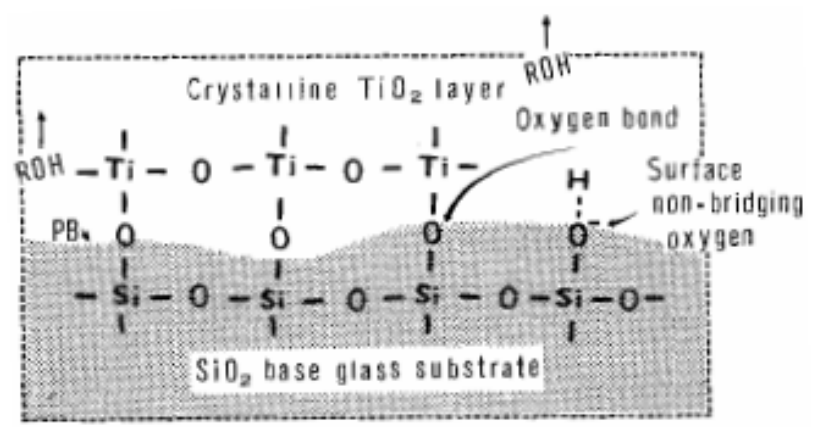

Abb. 5. Adhäsion des Metalloxid- $\left(\mathrm{TiO}_{2}\right)$ Films auf der Glasoberflche (Fallet et al., 2001).

Partikeln von 50 bis $125 \AA$ A Durchmesser, die allmählich zu einer geschlossenen Schicht zusammenwachsen.

Festes $\mathrm{Ag}_{2} \mathrm{~S}$ ist ein Halbleiter vom n-Typ. Sowohl in der $\alpha$ - als auch in der $\beta$-Phase sind neben den Elektronen auch die Ag-Ionen beweglich. $\alpha-\mathrm{Ag}_{2} \mathrm{~S}$ hat hauptsächlich eine ionische Leitfähigkeit, die stark von der Temperatur abhängt. $\mathrm{Ab}$ $177^{\circ} \mathrm{C}$, wenn also die $\beta$-Form vorliegt, ist die Leitfähigkeit vorwiegend elektronisch und nur noch schwach von der Temperatur abhängig. Zusätzlich ist die Leitfähigkeit von der Zusammensetzung abhängig. Je höher der Schwefelgehalt ist, desto niedriger ist die Leitfähigkeit (Gmelins Handbuch der anorganischen Chemie, 1973).

Nach Jostan et al. (1985) steigt der Kontaktwiderstand von Silber, wenn es mit einer 60 bis $100 \mathrm{~nm}$ dicken Silbersulfidschicht bedeckt ist, auf 10 bis $100 \mathrm{~m} \Omega$ an.

Die Abnahme der Leitfähigkeit durch $\mathrm{Ag}_{2} \mathrm{~S}$ und Fehlstellen in der metallischen Oberfläche könnten zur Folge haben, dass die Kontakte der mit Silber beschichteten Polyamidfäden unterbrochen werden und die Schirmdämpfungswirkung abnimmt.

Die Ausbildung von Silbersulfidschichten auf versilbertem Polyamid-Garn wurde rasterelektronenmikroskopisch untersucht (Abb. 3). Dabei zeigte sich, dass die gesamte Oberfläche mit Silbersulfid belegt ist, was an der strukturierten Oberfläche deutlich wird.

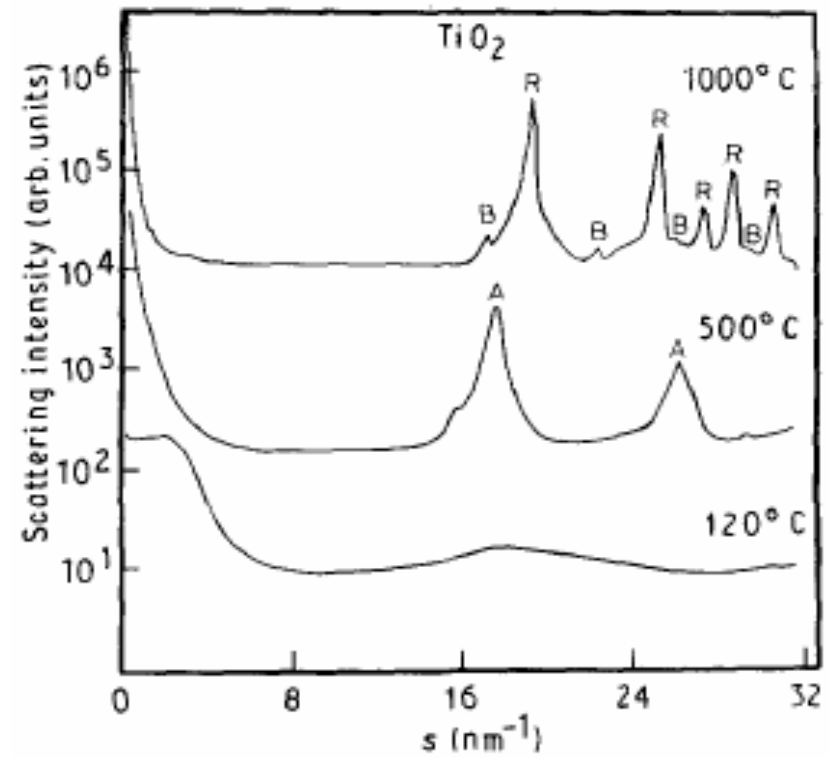

Abb. 6. Röntgendiffraktometrische Untersuchung von $\mathrm{TiO}_{2}$ nach einstündiger thermischer Behandlung A: Anatas, R: Rutil, B: Brookit (Samuneva et al., 1993).

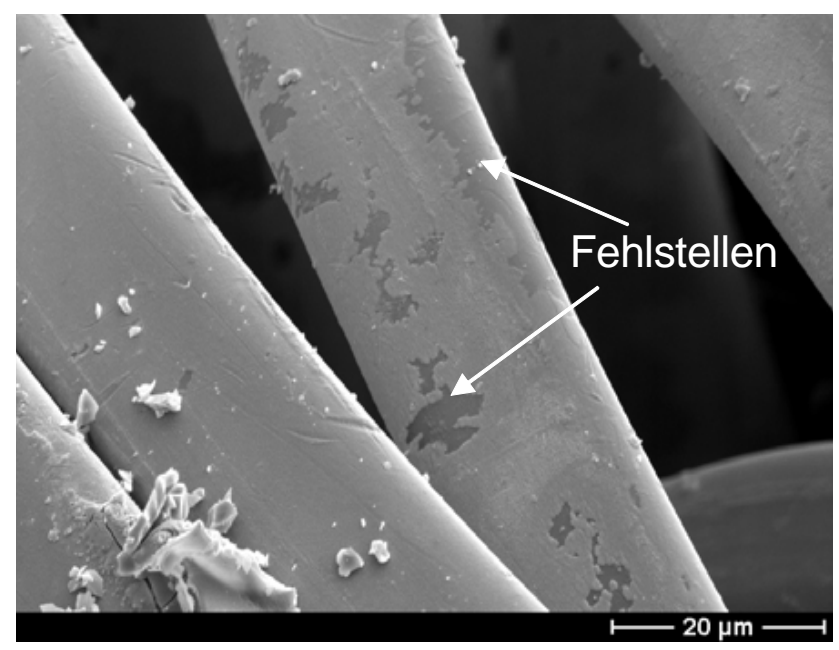

Abb. 7. Fehlstellen in $\mathrm{TiO}_{2}$-Schichten auf versilberten PolyamidGarn (REM-Aufnahmen).

\section{Herstellung definierter Silbersulfid-schichten}

Die Herstellung von Silbersulfid aus der Gasphase führt zu nicht reproduzierbaren Ergebnissen. Silbersulfidschichten lassen sich aber nasschemisch reproduzierbar herstellen.

Tuccillo und Nielsen beschrieben 1971 erstmals einen Test, um das Anlaufverhalten von dentalen Goldlegierungen zu untersuchen. Der Testaufbau bestand aus einem Kunststoffrad mit 8 Löchern, das sich mit $1 \mathrm{Upm}$ vertikal drehte. Als Beschichtungslösung setzten sie eine 0,5\%ige Natriumsulfidlösung ein (Tuccillo und Nielsen, 1971). 


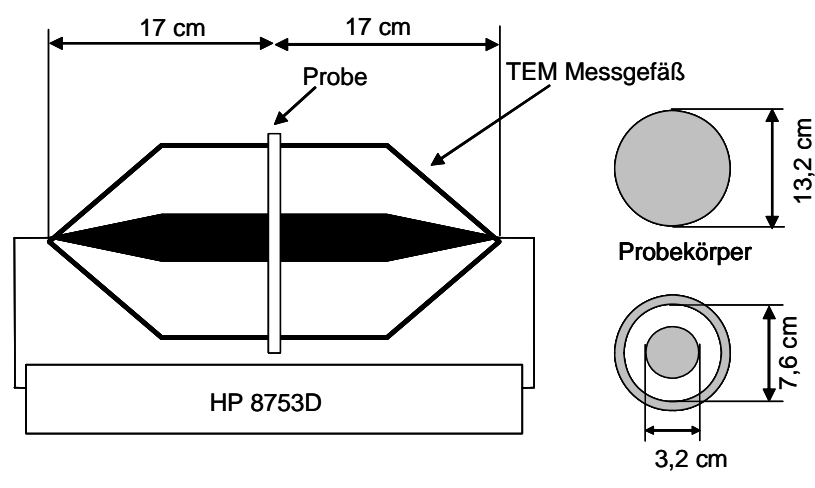

Referenzkörper

Abb. 8. Schematische Darstellung des Testaufbaus für die Schirmdämpfungsmessung nach ASTM D 4935-99 (1999).

Courty et al. (1991) untersuchten das Anlaufverhalten einer Goldlegierung mittels Tuccillo-Nielsen-Test. Die AnlaufExperimente wurden in einer Lösung aus $0,1 \mathrm{M} \mathrm{Na}_{2} \mathrm{~S}$ in $1 \mathrm{M}$

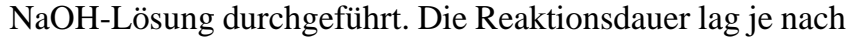
Versuch zwischen 0,5 und $5 \mathrm{~h}$. Die Autoren bestimmten die Zusammensetzung und die Dicke der entstandenen Oberflächenschichten durch Augerelektronenspektroskopie, Coulometrie und Röntgenbeugung. Eines der Ergebnisse war, dass die Anlaufrate im Tuccillo-Nielsen-Test eine lineare Funktion der Zeit ist (Courty et al., 1991).

1992 untersuchten Randin et al. (1992) unterschiedliche Anlauftests auf Reprodu-zierbarkeit sowie Stärke der Verfärbung. Unter den Versuchen waren zwei Gasphasentests (Schwefelblüten-Test, Thioacetamid-Test) und ein Flüssigphasen-test (Tuccillo-Nielsen-Test). Für den Flüssigphasentest wurden drei unter-schiedliche Lösungen untersucht:

\section{1. $0,26 \mathrm{M} \mathrm{Na}_{2} \mathrm{~S}, 3 \mathrm{~h}$ Reaktionsdauer}

\section{2. $0,1 \mathrm{M} \mathrm{Na}_{2} \mathrm{~S}, 8 \mathrm{~h}$ Reaktionsdauer}

\section{3. $0,1 \mathrm{M} \mathrm{Na}_{2} \mathrm{~S}+1 \mathrm{M} \mathrm{NaOH}, 3 \mathrm{~h}$ Reaktionsdauer}

Die Untersuchungen zeigen, dass das Anlaufverhalten durch den Flüssigphasentest besser als durch die GasphasenTests wiedergegeben wird, obwohl die Verfärbungen ungleichmäßiger waren. Bei den unterschiedlichen Lösungen gab es fast keinen Unterschied zwischen 1 und 3. Lösung 2 schnitt als schlechteste ab (Randin et al., 1992).

\section{$5 \quad$ Titandioxid}

In der Literatur Samuneva et al. (1993) wird von den antikorrosiven Eigenschaften keramischer Schichten, die durch das Sol-Gel-Verfahren hergestellt wurden, berichtet. Wenn der Angriff von korrosiven Medien durch eine keramische

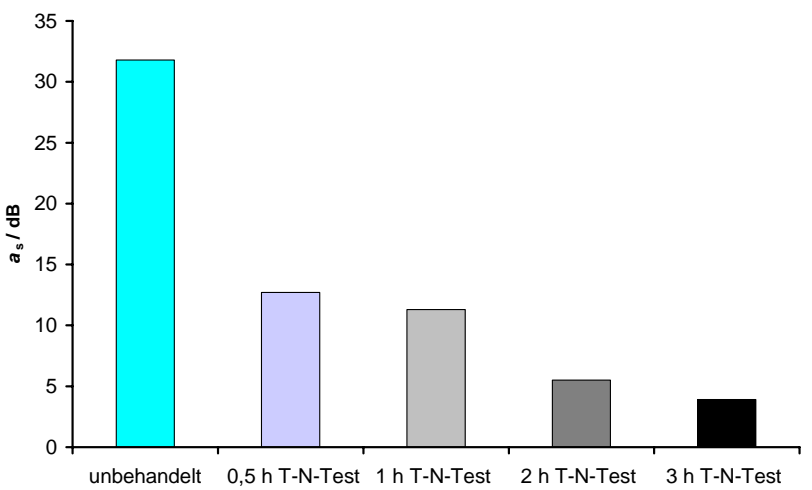

Abb. 9. Vergleich der Schirmdämpfung $\alpha_{S}$ eines silberbeschichteten Gestricks bei $900 \mathrm{MHz}$, unterschiedliche Reaktionszeiten der Tuccillo-Nielsen-Lösung.

Schicht aus Titandioxid $\left(\mathrm{TiO}_{2}\right)$ verhindert werden kann, lieBe sich vielleicht auch die Bildung von Silbersulfid auf der Silberschicht des Polyamid-Garns vermeiden.

Um die Silberschicht auf dem Polyamid-Garn vor Korrosion zu schützen, wurde es mittels Sol-Gel-Verfahren mit dem Halbleiter Titandioxid beschichtet. Die Reaktionen, die während der Sol-Gel-Beschichtung stattfinden, lassen sich schematisch wie folgt darstellen (Samuneva et al., 1993):

$\mathrm{Ti}(\mathrm{OR})_{4}+4 \mathrm{H}_{2} \mathrm{O} \rightarrow \mathrm{Ti}(\mathrm{OH})_{4}+4 \mathrm{ROH}$

$\mathrm{Ti}(\mathrm{OH})_{4} \stackrel{\mathrm{T}}{\longrightarrow} \mathrm{TiO}_{2}+2 \mathrm{H}_{2} \mathrm{O}$

Samuneva et al. (1993) untersuchten die strukturellen Charakteristika von dünnen $\mathrm{TiO}_{2}$-Schichten, die mittels Sol-GelVerfahren auf Glassubstraten aufgebracht wurden. In Abb. 5 ist die Bindung des Titandioxids auf der Glasoberfläche abgebildet.

Wurde die Probe bei $120^{\circ} \mathrm{C} 10 \mathrm{Min}$. getrocknet, konnte mittels Röntgendiffraktometrie keine kristalline Phase nachgewiesen werden. Das $\mathrm{TiO}_{2}$ liegt amorph vor. Anatas und Brookit sind metastabil und wandeln sich bei höheren Temperaturen in den stabilen Rutil um.

Elektronenbeugungsuntersuchungen der $\mathrm{TiO}_{2}$-Schichten zeigten auf, dass das röntgenamorphe $\mathrm{TiO}_{2}$ polykristallin vorliegt (Fallet et al., 2001).

Aus der rasterelektronischen Untersuchung eines versilberten Polyamid-Garns, welches mit $\mathrm{TiO}_{2}$ beschichtet wurde, ergab sich, dass die $\mathrm{TiO}_{2}-\mathrm{Schich}$ große Fehlstellen aufweist.

\section{Beseitigung von Silbersulfidschichten}

Eine Möglichkeit zur Beseitigung von Silbersulfidschichten ist die mechanische Reinigung (Polieren). Die Grundvoraussetzung hierfür ist jedoch ein guter Kontakt zwischen Poliergut und Poliermittel. Eine weitere Reinigungsmöglichkeit 


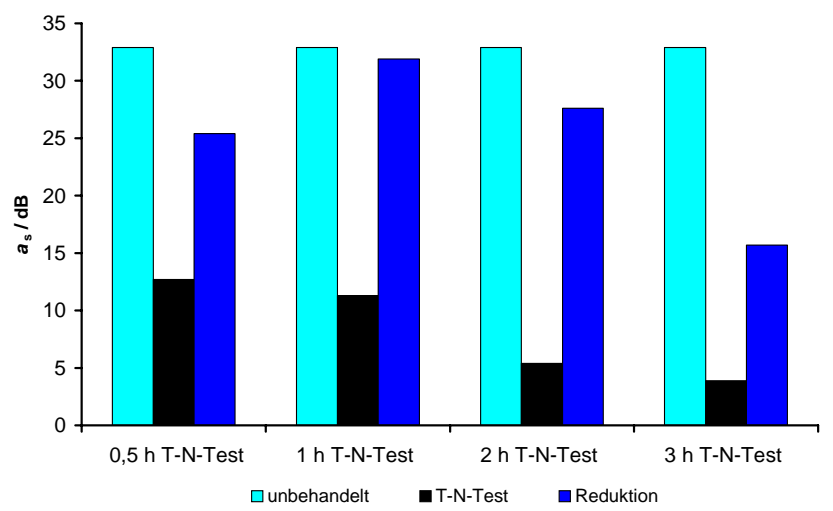

Abb. 10. Vergleich der Schirmdämpfung $\alpha_{s}$ eines versilberten Gestricks bei $900 \mathrm{MHz}$.

sind Tauchbäder, z.B. aus saurer Thioharnstofflösung. Für Polyamid ist dies nicht anwendbar, da sich Polyamid in sauren Medien auflöst.

Eine weitere Möglichkeit ist die Oxidation eines unedleren Metalls, z.B. Aluminium, und Reduktion des Silbersulfids zu elementarem Silber:

$2 \mathrm{Al}+3 \mathrm{Ag}_{2} \mathrm{~S} \rightarrow 2 \mathrm{Al}^{3+}+3 \mathrm{~S}^{2-}+6 \mathrm{Ag}$

Für diesen Versuch wurden die Proben in Aluminiumfolie gewickelt und in einer $150 \mathrm{~g} \mathrm{~L}^{-1}$ Natriumchloridlösung bei $60^{\circ} \mathrm{C} 1 \mathrm{~h}$ gelagert, anschließend mit je 3,1 destilliertem Wasser gewaschen und im Abzug über Nacht getrocknet.

\section{Schirmdämpfungsmessungen nach ASTM D 4935-99}

Zur Durchführung der Messungen wurde ein TEMMessgefäß (Wandel \& Goltermann) verwendet.

Bei der Messanordnung wurde das zu prüfende Material durch die koaxiale TEM-Welle gleichmäßig mit allen Polarisationen gemessen. Der Frequenzbereich der Messungen lag zwischen $300 \mathrm{MHz}$ und $4 \mathrm{GHz}$.

Die HF-Strahlung wurde mit dem Netzwerkanalysator HP 8753D (Hewlett Packard, Palo Alto, Kalifornien) erzeugt und gemessen.

Die voneinander getrennten Innen- und Außenleiter des Messgefäßes der Firma Wandel \& Goltermann bestehen aus Messing. Der Innenleiter ist zusätzlich mit einer Teflonstütze ausgerüstet.

In Abb. 9 ist der Vergleich der Schirmdämpfungswerte $a_{s}$ eines versilberten Gestricks nach unterschiedlichen Reaktionszeiten beim Tuccillo-Nielsen-Test (T-N-Test) dargestellt.

Die Schirmdämpfung sinkt mit steigender Einwirkdauer der Tuccillo-Nielsen-Lösung auf das silberbeschichtete Polyamid-Garn auf ca. $13 \%$ des ursprünglichen Schirmdämpfungswerts.

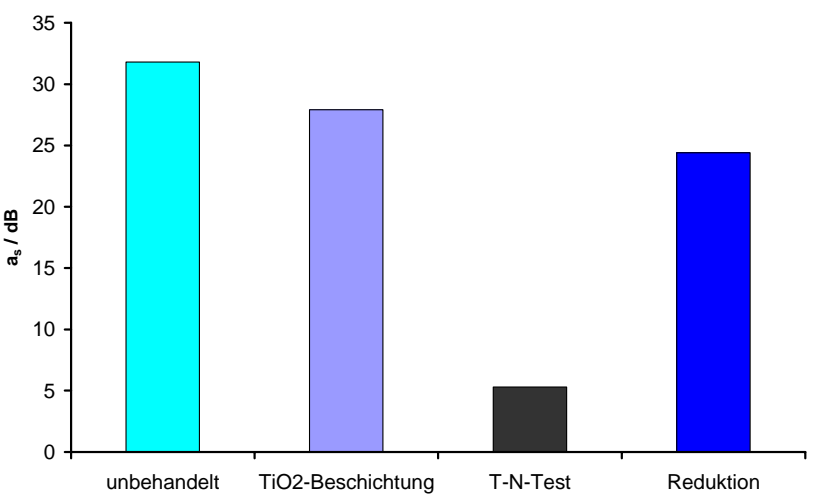

Abb. 11. Vergleich der Schirmdämpfung $\alpha_{S}$ eines versilberten Gestricks bei $900 \mathrm{MHz}$.

\section{Ergebnisse}

In Abb. 10 sind die unterschiedlichen Schirmdämpfungswerte $a_{s}$ eines versilberten Gestricks bei $900 \mathrm{MHz}$ dargestellt. Es ist der Vergleich zwischen dem Original (unbehandelt), nach unterschiedlichen Reaktionszeiten des silberbeschichteten Gestricks beim Tuccillo-Nielsen-Test (T-N-Test) sowie anschließender Reduktion der Silbersulfidschicht abgebildet.

Mit steigender Einwirkdauer der Tuccillo-Nielsen-Lösung sinkt die Schirmdämpfung. Die Reduktion der Silbersulfidschicht mit Aluminiumfolie in Natriumchlorid-Lösung führt zu schwankenden Ergebnissen. Ein kritischer Parameter ist hierbei die wirksame Kontaktfläche zwischen dem Silbersulfid und dem Aluminium (heterogene Reaktion).

In Abb. 11 sind die unterschiedlichen Schirmdämpfungswerte $a_{s}$ eines versilberten Gestricks bei $900 \mathrm{MHz}$ dargestellt. Es ist der Vergleich zwischen einem versilberten Original-Gestrick (unbehandelt) und einem zusätzlich mit Titandioxid $\left(\mathrm{TiO}_{2}\right)$ beschichteten Gestrick $\mathrm{zu}$ sehen. Außerdem sind die Schirmdämpfungswerte des $\mathrm{TiO}_{2}$-beschichteten Gestricks nach dreistündigem TuccilloNielsen-Test (T-N-Test) sowie anschließender Reduktion der Silbersulfidschicht abgebildet.

\section{Zusammenfassung}

Die Schirmdämpfungsmessungen zeigten, dass die untersuchten versilberten Gestricke eine weitgehende frequenzunabhängige Schirmdämpfung von ca. $40 \mathrm{~dB}$ aufweisen. Durch eine kontrollierte Belegung der Oberfläche mit Silbersulfid mit Hilfe eines modifizierten Tuccillo-Nielsen-Tests sank die Schirmdämpfung auf ca. 5-10 dB ab. Die im Rahmen dieser Arbeit untersuchte Titandioxid-Beschichtung, welche durch ein Sol-Gel-Verfahren auf das versilberte Gestrick aufgebracht wurde, erwies sich als fast wirkungslos.

Die Reduktion der Silbersulfidschicht durch Aluminium führt nicht zum Ausgangswert der Schirmdämpfung, was 
möglicherweise auf den ungenügenden Kontakt zwischen dem Silbersulfid und dem Aluminium zurückzuführen ist.

Die Ergebnisse dieser Untersuchungen sind noch als vorläufig zu betrachten, da aus Zeitgründen nur Einzelbestimmungen durchgeführt werden konnten.

Festzuhalten bleibt jedenfalls, dass die Schirmdämpfung versilberter Gestricke durch Silbersulfidbildung erheblich beeinträchtigt werden kann.

Danksagung. Für ihre umfangreiche experimentelle Unterstützung danken die Autoren Prof. Dr.-Ing. M. Koch und Dipl.-Ing. T. Frenzel (Leibniz Universität Hannover, Inst. für Grundlagen der Elektrotechnik und Messtechnik, Fachgebiet Elektromagnetische Verträglichkeit) sowie Prof. Dipl.-Ing. P. Pauli (Universität der Bundeswehr München, Wissenschaftliche Einrichtung für Hochfrequenztechnik und Nachrichtenübertragungstechnik).

\section{Literatur}

ASTM Designation: D 4935-99, Standard Test Method for Measuring the Electromagnetic Shielding Effectiveness of Planar Materials American Society for Testing and Materials, Annual Book of ASTM Standards, 1999.

Courty, H., Mathieu, H. J. and Landolt, D.: Tarnishing of Au-Ag$\mathrm{Cu}$ alloy studied by Auger electron spectroscopy and coulometry, Werkstoffe und Korrosion, VCH Verlagsgesellschaft mbH, Weinheim, 42. Jahrgang, 288-295, 1991.
Fallet M., Mahdjoub, H., Gautier, B., and Bauer, J.-P.: Electrochemical behavior of ceramic sol-gel coatings on mild steel, Journal of Non-Crystalline Solids, 527-533, 2001.

Gmelins Handbuch der anorganischen Chemie: Silber, Teil A 3, Chemisches Verhalten, Nachweis und Bestimmung. Toxicitt, Kolloides Silber Verlag Chemie GmbH, Weinheim Bergstrasse, 1971.

Gmelins Handbuch der anorganischen Chemie: Silber, Teil B 3, Verbindungen mit Schwefel, Selen, Tellur, Polonium, Bor, Kohlenstoff und Silicium Verlag Chemie GmbH, Weinheim Bergstrasse, 1973.

Jostan, J. L., Mussinger, W. und Bogenschtz, A. F.: Langzeitprfungen an Elektrowerkstoffen, Metalloberflche, 39, 135-137, 1985.

Kim, H.: Corrosion process of silver in environments containing $0,1 \mathrm{ppm} \mathrm{H}_{2} \mathrm{~S}$ and $1,2 \mathrm{ppm} \mathrm{NO}_{2}$ Materials and Corrosion 54, Wiley-VCH Verlag GmbH \& Co. KgaA, Weinheim, 243-250, 2003.

Randin, J. P., Renaud J. P., and Ramoni, P.: Tarnishing of $\mathrm{AuAgCu}$ alloys. Choice of an accelerated test, Werkstoffe und Korrosion, VCH Verlagsgesellschaft mbH, Weinheim, 43. Jahrgang, 48-55, 1992.

Samuneva, B., Kazhukharov, V.; Trapalis, Ch.; Kranold, R.: Sol-gel processing of titanium-containing thin coatings, Part I. Preparation and structure, Journal of Materials Science, 28, 9, 2352-2360, 1993.

Tuccillo, J. J. and Nielsen, J. P.: Observations of onset of sulfide tarnish on gold-base alloys, J. Prosth. Dent., 25, 6, 629-637, 1971. 